\title{
Sorting nexin 10 controls mTOR activation through regulating amino-acid metabolism in colorectal cancer
}

\author{
Yunchen Le', Sulin Zhang ${ }^{1}$, Jiahui Ni ${ }^{1}$, Yan You' ${ }^{1}$ Kejing Luo', Yunqiu Yu ${ }^{1}$ and Xiaoyan Shen ${ }^{1}$
}

\begin{abstract}
Amino-acid metabolism plays a vital role in mammalian target of rapamycin (mTOR) signaling, which is the pivot in colorectal cancer (CRC). Upregulated chaperone-mediated autophagy (CMA) activity contributes to the regulation of metabolism in cancer cells. Previously, we found that sorting nexin 10 (SNX10) is a critical regulator in CMA activation. Here we investigated the role of SNX10 in regulating amino-acid metabolism and mTOR signaling pathway activation, as well as the impact on the tumor progression of mouse CRC. Our results showed that SNX10 deficiency promoted colorectal tumorigenesis in male FVB mice and CRC cell proliferation and survival. Metabolic pathway analysis of gas chromatography-mass spectrometry (GC-MS) data revealed unique changes of amino-acid metabolism by SNX10 deficiency. In HCT116 cells, SNX10 knockout resulted in the increase of CMA and mTOR activation, which could be abolished by chloroquine treatment or reversed by SNX10 overexpression. By small RNA interference (siRNA), we found that the activation of mTOR was dependent on lysosomal-associated membrane protein type-2A (LAMP-2A), which is a limiting factor of CMA. Similar results were also found in Caco-2 and SW480 cells. Ultra-high-performance liquid chromatography-quadrupole time of flight (UHPLC-QTOF) and GC-MS-based untargeted metabolomics revealed that 10 amino-acid metabolism in SNX10-deficient cells were significantly upregulated, which could be restored by LAMP-2A siRNA. All of these amino acids were previously reported to be involved in mTOR activation. In conclusion, this work revealed that SNX10 controls mTOR activation through regulating CMA-dependent amino-acid metabolism, which provides potential target and strategy for treating CRC.
\end{abstract}

\section{Introduction}

Colorectal cancer (CRC) is the third most commonly diagnosed cancer in males and the second in females, with an estimated 1.4 million cases and 693900 deaths occurring globally in $2012^{1}$. Current treatment approaches are not sufficient ${ }^{2}$. Only $40 \%$ of CRC are diagnosed at early localized stage and the 5 -year survival is $13 \%$ when the disease has spread to distant organs ${ }^{3}$. The discovery of new therapeutic strategies in CRC treatment is an urgent need and has attracted a great deal of research interest ${ }^{4-6}$. Although cancer is widely known as a genetic disease,

Correspondence: Yunqiu Yu (yqyu@shmu.edu.cn) or Xiaoyan Shen (shxiaoy@fudan.edu.cn)

${ }^{1}$ School of Pharmacy, Fudan University, Shanghai 201203, PR China Edited by GM Fimia emerging evidence indicates that cancer is primarily a metabolic disease involving metabolism reprogramming such as Warburg effect ${ }^{7}$. Recently, metabolomics, an "omics" science aim to comprehensive profile metabolism in organism using cutting-edge analytical chemistry techniques and advanced computational methods, is being used to explore disease mechanisms and to identify novel drug targets ${ }^{8}$. In various bio-fluids and tissues of CRC patients, metabolomics studies have uncovered multiple altered metabolites in cellular respiration/carbohydrate, lipid, amino-acid, nucleotide, and other significant metabolite perturbations ${ }^{9-11}$. However, the understanding of the biological significance underlying the metabolism alternation is limited. Further study of the represented molecular mechanism of metabolism 


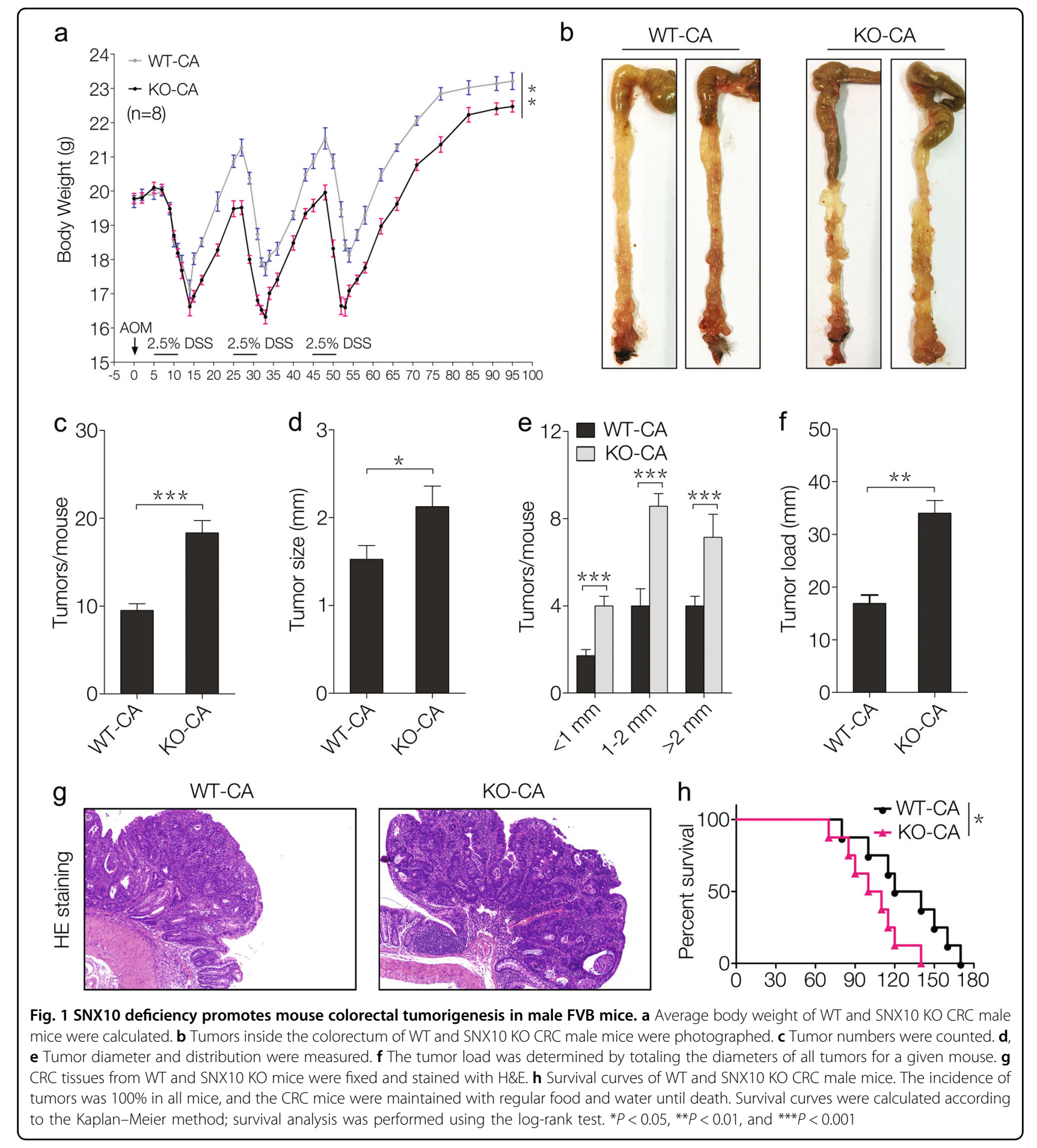

imbalance and its impact on CRC development will help to uncover new targets to treat CRC.

Sorting nexin 10 (SNX10) is one of the simplest structure members of sorting nexins (SNXs), a family of evolutionarily conserved proteins containing the phox-homology domain, which targets SNXs to endosome membrane through bind with phosphoinositide to regulate endosomal cargo sorting and trafficking ${ }^{12}$. An important way for the endosomes to participate in cell catabolism is lysosome-dependent autophagy. Autophagy has significant effects on cancer cell survival and may play dual role in carcinogenesis ${ }^{13}$. Chaperone-mediated autophagy (CMA), one of the three 
types of autophagy, is responsible to deliver the cytosolic proteins with a specific pentapeptide motif to be degraded in lysosomes ${ }^{14}$. The activation of CMA has been implicated in promoting lung cancer cell survival ${ }^{15}$. High level of CMA had been observed in large number of cancer cell lines and directly linked to tumor glycolysis, and pyruvate and fatty acid metabolism ${ }^{16}$. Thus, cancer cells may use CMA to rewrite the cellular metabolic homeostasis to facilitate tumor proliferation. Recently, we found that SNX10 controls CMA activity by mediating the maturation of cathepsin A, a key enzyme involved in lysosomal-associated membrane protein-2A (LAMP-2A) degradation, and plays an essential role in alcohol-induced liver injury and steatosis ${ }^{17}$. Through regulating chaperone-mediated autophagic a
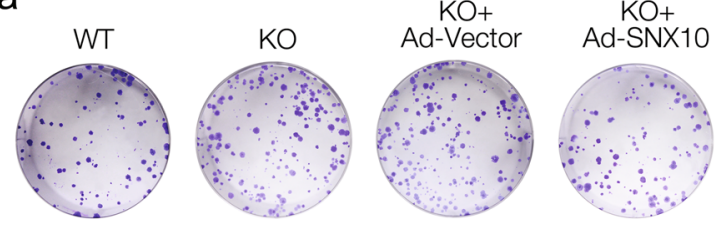

$\mathrm{KO}+$

C

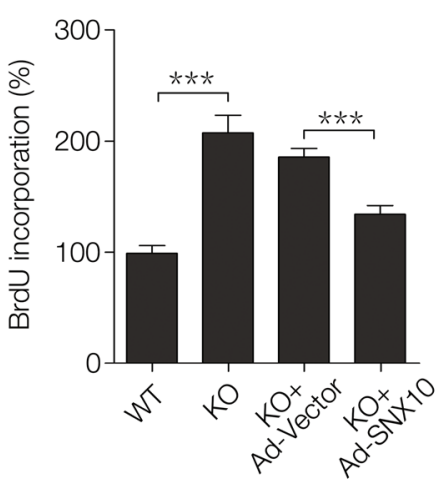

e

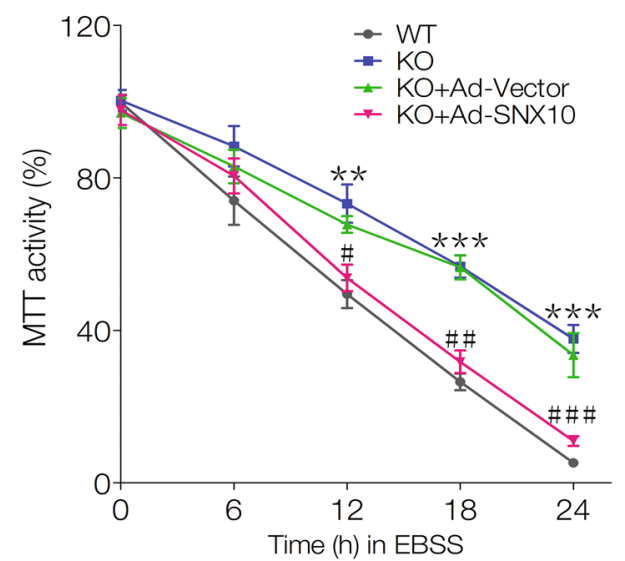

d

\section{b}
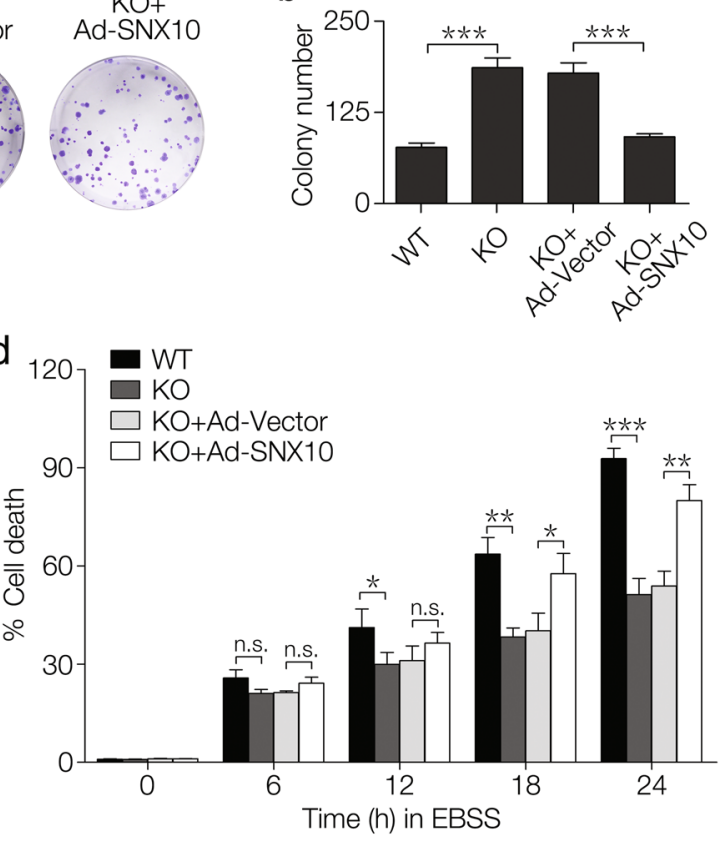

f

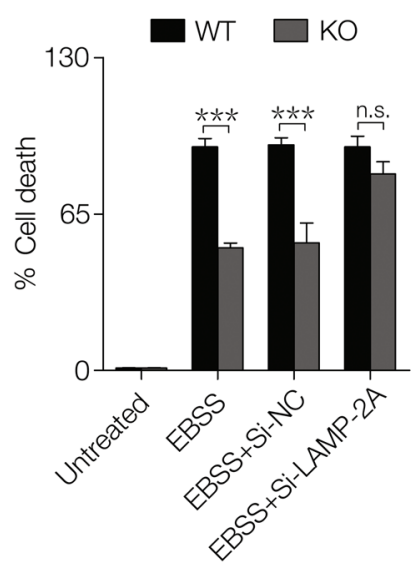

Fig. 2 SNX10 deficiency facilitates HCT116 cell proliferation and survival under nutritional deprivation. a The colony-forming ability of WT, SXN10 KO HCT116 cells, and SXN10 KO HCT116 cells infected with Ad-vector or Ad-SNX10. b Quantification of colony numbers in a. c The proliferation of WT, SXN10 KO HCT116 cells, and SXN10 KO HCT116 cells infected with Ad-vector or Ad-SNX10 was measured by BrdU cell proliferation assay. d, e WT, SXN10 KO HCT116 cells, and SXN10 KO HCT116 cells infected with Ad-vector or Ad-SNX10 were cultured in EBSS for 0, 6, 12,18 , or $24 \mathrm{~h}$. $\mathbf{d}$ The percentage of dead cells was determined by trypan blue exclusion assay at the indicated time points. e The cell viability was determined by MTT assay at the indicated time points. $\mathbf{f W T}$ and SXN10 KO HCT116 cells were untreated, or were treated with EBSS for $24 \mathrm{~h}$, or were transfected with nontargeting siRNA (Si-NC) or LAMP-2A siRNA (Si-LAMP-2A), followed by EBSS treatment for $24 \mathrm{~h}$, and the percentage of dead cells was determined by trypan blue exclusion assay. Data are derived from at least three independent experiments and represented as means \pm SEM in the bar graphs. Not significant (n.s.), ${ }^{*} P<0.05,{ }^{* *} P<0.01$, and ${ }^{* * *} P<0.001$ 
degradation of $\mathrm{p} 21^{\mathrm{Cip} 1 / \mathrm{WAF} 1}$, SNX10 acts as a tumor suppressor in tumorigenesis and progression of colorectal cancer $^{18}$. These results encouraged us to explore the possible role of SNX10 in metabolism reprogramming through modulating CMA activation. In the present study, ultrahigh-performance liquid chromatography-quadrupole time of flight (UHPLC-QTOF)- and gas chromatography-mass spectrometry (GC-MS)-based untargeted metabolomics were used to analyze the metabolism alterations in the tumor tissues of wild-type (WT) and SNX10 knockout (KO) CRC male FVB mice, and the relation of amino-acid metabolism alteration and CRC cell proliferation and survival was investigated.

\section{Results}

\section{SNX10 deficiency promotes colorectal tumorigenesis in male FVB mice}

To evaluate the possible role of SNX10 on tumorigenesis of CRC, we generated an azoxymethane $(\mathrm{AOM}) /$ dextran sulfate sodium (DSS)-induced CRC model in male FVB mice. SNX10 KO CRC mice had more weight loss and recovered more slowly during each DSS exposure compared with WT mice (Fig. 1a). The mice were sacrificed on day 95 following induction, the incidence of tumors was $100 \%$ in all mice. However, in SNX10 KO mice the number of tumors increased almost two times and the tumor sizes were larger compared to WT mice (Fig. 1b-e). Correspondingly, the tumor load, which represents the sum of the diameters of all tumors in a given mouse, was significantly increased in SNX10 KO mice (Fig. 1f). These results were also confirmed by histological assessment (Fig. 1g). Moreover, SNX10 KO CRC mice showed poorer survival (Fig. 1h). These results indicate that SNX10 deficiency significantly promoted tumorigenesis of CRC in male FVB mice.

\section{SNX10 deficiency facilitates HCT116 cell proliferation and survival under nutritional deprivation}

To further confirm the effect of SNX10 deficiency on CRC, we first measured the expression of SNX10 mRNA in NCM460 cell, which is a normal human colon mucosal epithelial cell line, and in three CRC cell lines HCT116, Caco-2, and SW480 cells (supplementary Fig. S1), and found that SNX10 mRNA level was decreased in CRC cells. We then compared the proliferation and survival ability between WT and SNX10 KO HCT116 cells. The colony numbers of SNX10 KO HCT116 cells were more than two times of WT cells, while SNX10 reintroduction could partially reversed this result (Fig. 2a, b). BrdU cell proliferation assay also showed that cell proliferation ability was enhanced by SNX10 deficiency and this effect was partially reversed by SNX10 reintroduction (Fig. 2c). To explore whether
SNX10 is involved in CRC cell survival under nutritional deprivation, WT and SNX10 KO HCT116 cells were cultured in Earles balanced salt solution (EBSS). SNX10 KO cells showed more resistance to trypan blue staining than WT cells, and this effect could be partially reversed by SNX10 reintroduction (Fig. 2d), suggesting SNX10 deficiency facilitated CRC cell survival under nutritional deprivation. MTT assay also confirmed the impact of SNX10 deficiency on CRC cell survival under nutritional deprivation (Fig. 2e). Moreover, under nutritional deprivation, the difference of cell survival ability between WT and SNX10 KO HCT116 cells could be abolished by LAMP-2A depletion (Fig. 2f). LAMP-2A is the rate-limiting factor for CMA degradation. The level of LAMP-2A protein in the lysosomal membrane directly correlated with CMA activity. The results also indicate that SNX10 may be involved in CMA-mediated degradation. These results were validated in Caco- 2 and SW480 cells (supplementary Fig. S2).

\section{Untargeted metabolomics reveals significant metabolism alternations in tumor tissues of CRC male mice caused by SNX10 KO}

In order to investigate the metabolic alternations caused by SNX10 KO, untargeted metabolomics was applied on the colonic tumor tissues of male FVB mice. Liquid chromatography-MS (LC-MS)- and GC-MS-based analysis platforms were both used due to their different metabolite coverage. UHPLC-QTOF positive ion-mode analysis generated 8662 features (Fig. 3a). The retention time drifts of quality control (QC) samples were $<0.2 \mathrm{~min}$, which indicated good reproducibility of our methodology in this analysis mode (Fig. 3b). There were 835 positive ion features distributed in the $\mathrm{m} / \mathrm{z}$ range from 50 to 1000 and retention time from 9.5 to $1619.7 \mathrm{~s}$ to be significantly changed $(P<0.05$; Fig. $3 c)$. Multivariable statistical analysis models were applied in order to obtain overview of the metabolome differences among the samples. Partial least squares-discriminant analysis (PLS-DA) score plot (Fig. 3d) and principal component analysis (PCA) model plot (Fig. 3e) provided the separation patterns among the most significant principal components. They showed a complete separation of WT and KO samples. Crossvalidation bar graphs and details that used to evaluate the common performance of PLS-DA model were shown in supplementary Fig. S3. A total of 184 important features were selected by volcano plot (Fig. 3f) with fold change (FC) test threshold 2 and $t$-test threshold 0.05 . The red dots represent features above the threshold. Both FCs and $P$ values were $\log$-transformed. The summary of features and metabolite numbers analyzed by untargeted metabolomics was shown in Table 1. The plots of negative ionmode UHPLC-QTOF and GC-MS analysis were shown in supplementary Fig. S4-S5. 

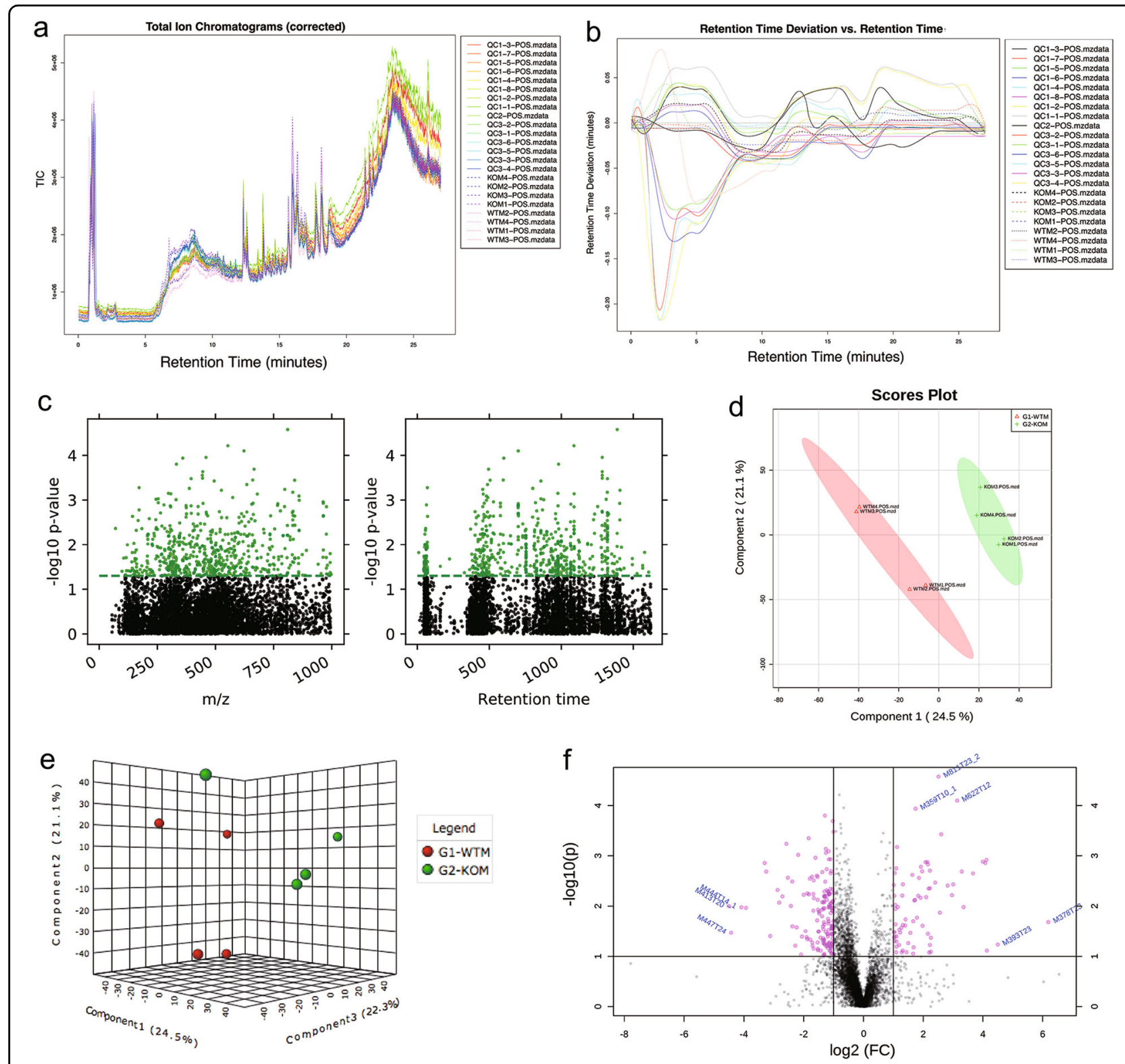

Fig. 3 Untargeted metabolomics and multivariate statistical analysis of tumor tissues from CRC male mice using UHPLC-QTOF in positive ion mode disclosed significant metabolic alternations caused by SNX10 deficiency. a Total ion chromatograms of WT (WTM), SNX10 KO (KOM), and quality control samples (QC). b Retention time deviation of all samples. c Manhattan map showed the distribution of significant features $(P<$ 0.05, green dots) in $\mathrm{m} / \mathrm{z}$ and retention time. $\mathbf{d}$ 2D score plot between the selected PCs of PLS-DA model. e 3D score plot between the selected PCs of PCA model. $\mathbf{f}$ Volcano plot with fold change threshold and $t$-test threshold to select the important features

\section{Carbohydrate, amino-acid, and nucleotide metabolism were upregulated by SNX10 deficiency}

UHPLC-QTOF analysis raw data were processed to generate the active metabolic pathway networks (Fig. 4a). Negative ion-mode analysis demonstrated that carbohydrate, amino-acid, and nucleotide metabolism were upregulated. The active metabolic pathways included glycolysis, pyruvate metabolism, and tricarboxylic acid (TCA) cycle suggested that glucose uptake and biosynthesis using intermediate metabolites in TCA cycle were increased in SNX10 KO mice. Purine metabolism was upregulated accompanied by the active pentose phosphate pathway. Sharply elevated fructose and galactose metabolism in CRC indicate that they were important energy resources for the CRC tumor proliferation. Positive ionmode analysis showed that purine metabolism, inositol phosphate metabolism, and starch metabolism were elevated in SNX10 KO tissues (supplementary Fig. S6).

Metabolic pathway analysis of GC-MS data revealed unique changes of amino-acid metabolism in SNX10- 
Table 1 The summary of features and metabolite numbers analyzed by untargeted metabolomics

\begin{tabular}{llll}
\hline & UHPLC-MS & & Negative ion mode \\
\cline { 2 - 3 } & Positive ion mode & 2570 \\
Number of peaks in raw data & 8662 & 1481 & 774 \\
Number of peaks after data pre-processing & 5140 & 437 & 773 \\
Number of significant features & 835 & 50 & 117 \\
Number of identified metabolites & 34 & 24 \\
\hline
\end{tabular}

deficiency mice. A set of amino-acid metabolism and aminoacyl-tRNA biosynthesis were upregulated in SNX10 KO tissue samples (Fig. 4b). GC-MS data-based heatmap showed an important concentration change of metabolites in SNX10 KO mouse tissues compared to WT tissues (Fig. 4c). The red blocks represented increased concentrations of amino acids all associated with $\mathrm{KO}$ samples. All the metabolite information generated from untargeted metabolomics were listed in supplementary Table S1-S3. Among them, we paid special attention to the amino-acid metabolism because it has been closely connected with mTOR signaling activation and tumor development.

\section{Over-activation of CMA caused by SNX10 deficiency upregulates mTOR signaling activation}

Due to the protein degradation through CMA can produce a large amount of cellular free amino acids, we then determined the possible correlation of increased CMA induced by SNX10 KO and amino-acid metabolism. LAMP-2A is an important limiting factor in CMA pathway. The expression of LAMP-2A in SNX10 KO cells increased dramatically under both normal and starvation conditions (Fig. 5a, b). Glyceraldehyde-3-phosphate dehydrogenase (GAPDH), a CMA substrate, negatively correlated with the expression of LAMP-2A and they highly overlapped in the cellular compartments (Fig. 5a, b). The upregulation of LAMP-2A and decrease of GAPDH in KO cells was suppressed by chloroquine (CQ), which is an autophagy inhibitor (Fig. 5c), or by SNX10 overexpression (Fig. 5d). The phosphorylated mTOR (pmTOR) levels were upregulated by SNX10 deficiency and positively correlated with LAMP-2A (Fig. 5a, c); while decreased when SNX10 was reintroduced or LAMP-2A was interfered (Fig. 5d, e). These results indicate that CMA process was implicated in the mTOR activation induced by SNX10 deficiency.

\section{Cellular accumulation of amino acid caused by SNX10 KO was dependent on LAMP-2A}

To confirm the relationship of CMA and mTOR activation, LAMP-2A siRNA was used to impair CMA activity. As shown in Fig. 6a, p-mTOR levels were elevated in
SNX10 KO cells and decreased when LAMP-2A was interfered. Selective ion mode GC-MS-based targeted metabolomics was used to measure relative concentrations of 14 amino acids, which were observed in untargeted metabolomics or identified as important mTORC1 activators in previous study in WT, KO, and LAMP-2A siRNA cells (Fig. 6a) ${ }^{19}$. The results showed that 13 amino acids, including Val, Leu, Ile, Pro, Gly, Ser, Thr, Asp, Met, Asn, Glu, Phe, and Arg were changed by SNX10 KO and most of them were increased except Glu, Phe, and Arg (Fig. 6b). After LAMP-2A siRNA, the elevated amino acids induced by SNX10 KO were decreased. The represented chromatograph of these amino acids was shown in Fig. 6c. We then analyzed the Pearson correlation coefficients between these amino acids and levels of LAMP-2A and p-mTOR. The results showed that the concentration of Val, Leu, Ile, Pro, Gly, Ser, Thr, Asp, Met, and Asn had great correlation with LAMP-2A and p-mTOR (Fig. 6d).

The relative values of amino-acid concentrations were obtained by comparison with internal standard (ISTD) tridecanoic acid. The added accurate weight of tridecanoic acid was $24 \mu \mathrm{g}$ for each sample. Results showed that glycine had the highest cellular level while asparagine concentration was the lowest among the analyzed amino acids. Due to the different ionization levels and response factors of these amino acids, the absolute cellular concentrations of these amino acids could not be directly calculated by the relative coefficient. Nevertheless, these results are sufficient to illustrate the amino-acid concentration differences between groups.

\section{Discussion}

In the present study, we investigated the comprehensive metabolic alternations in SNX10-deficient CRC male FVB mice using untargeted metabolomics analysis, and explored its regulatory mechanism underlying increased amino-acid metabolism in SNX10 KO cells and its impact on CRC cell proliferation and survival. Our metabolomics characterization of SNX10 KO CRC male mice is consistent with the hallmarks of cancer metabolism ${ }^{20}$, suggesting that the accumulative amino acids caused by SNX10 KO have profound effect on the CRC cell growth 


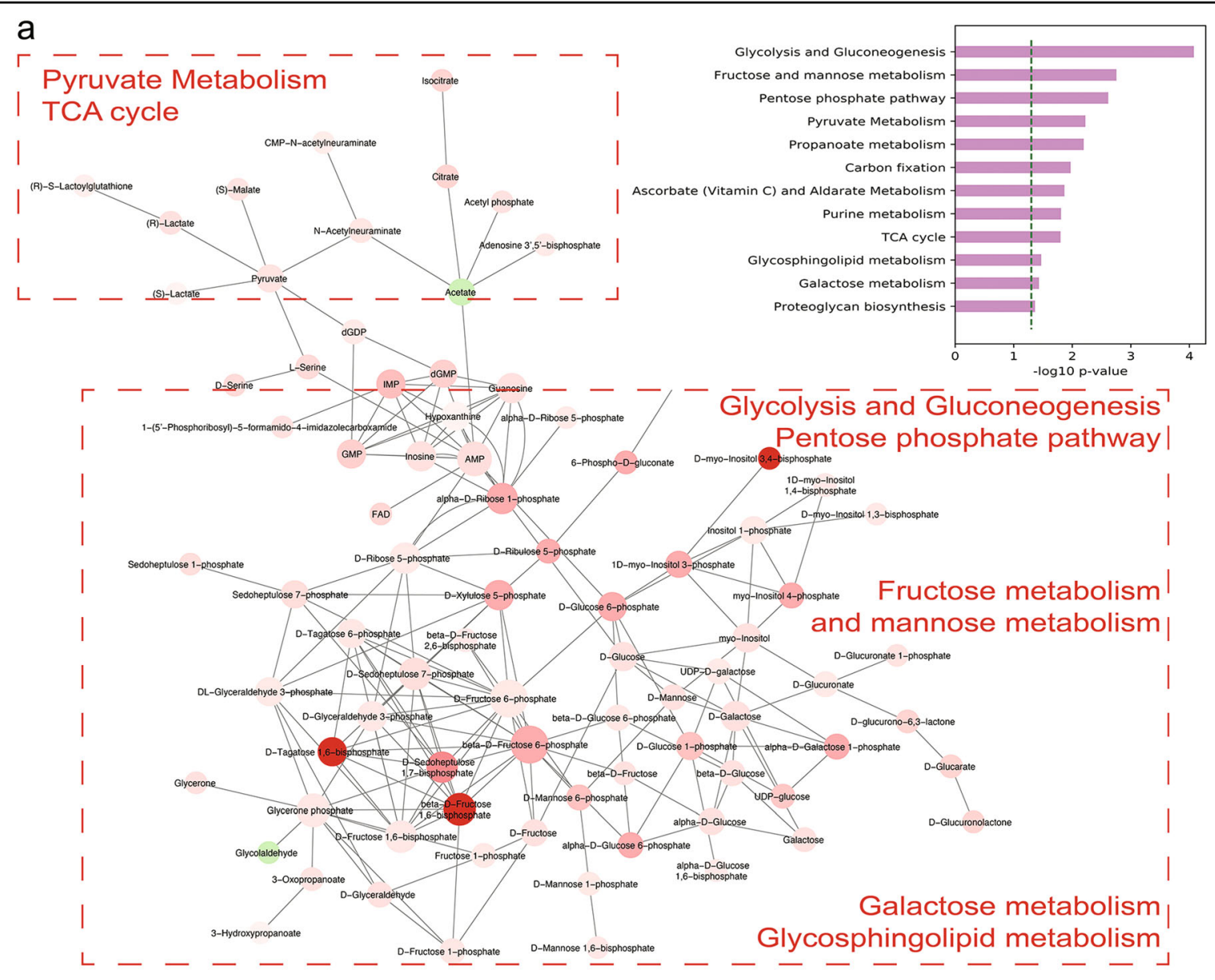

b
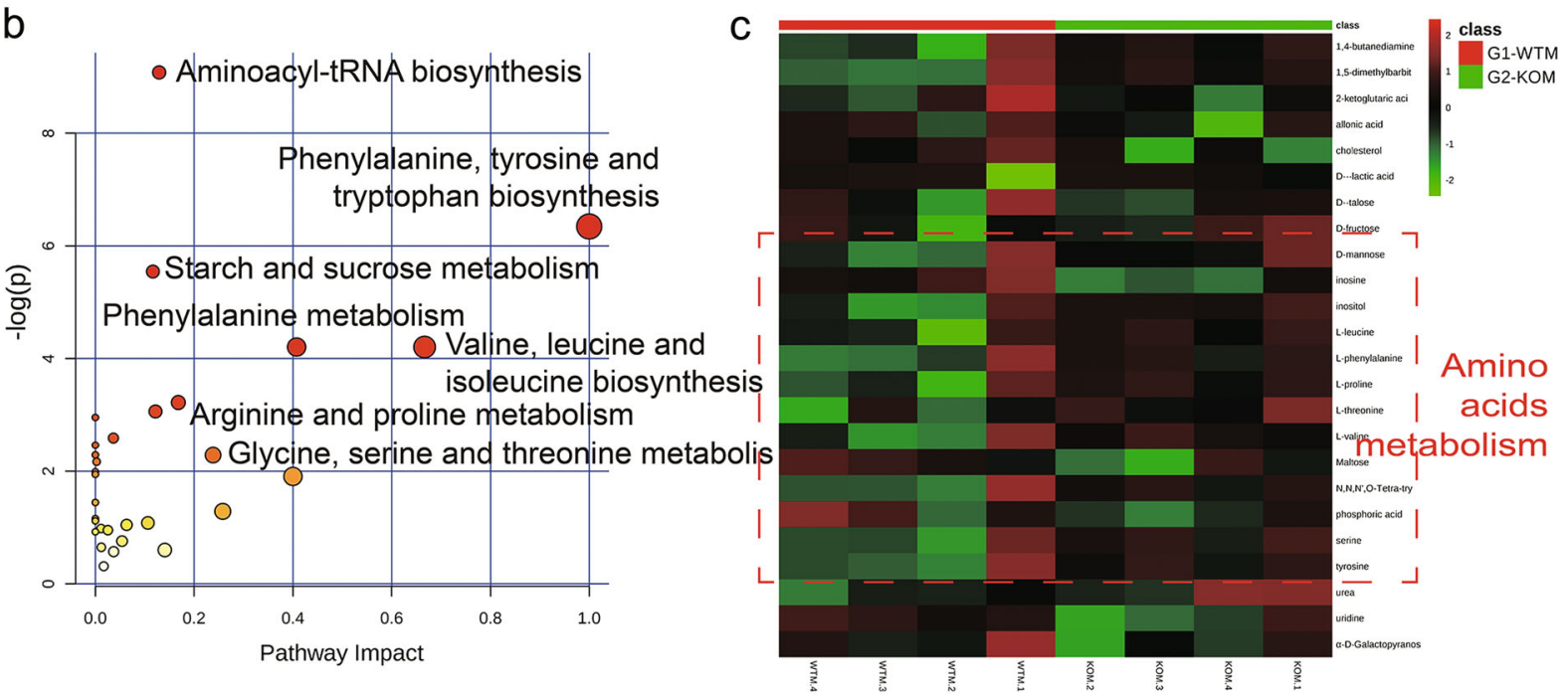

Fig. 4 Metabolic pathway networks based on the untargeted metabolomics using UHPLC-QTOF in negative ion mode and GC-MS analysis. a Metabolic networks of activity pathways that based on the UHPLC-QTOF negative ion-mode analysis and generated from software Mummichog and visualized via Cytospace. Metabolites are colored green (concentration decrease) or red (concentration increase), the size and color intensity represent the connectivity of the metabolite in the networks and magnitude of fold change. $\mathbf{b}$ The metabolome view that based on the GC-MS analysis and generated from the web-based metabolomics analysis tool MetaboAnalyst. All matched pathways according to $P$ values from pathway enrichment analysis and pathway impact values from pathway topology analysis. c Heatmap of the significant metabolites analyzed by GC-MS showed their concentration changing patterns across WT and KO samples 

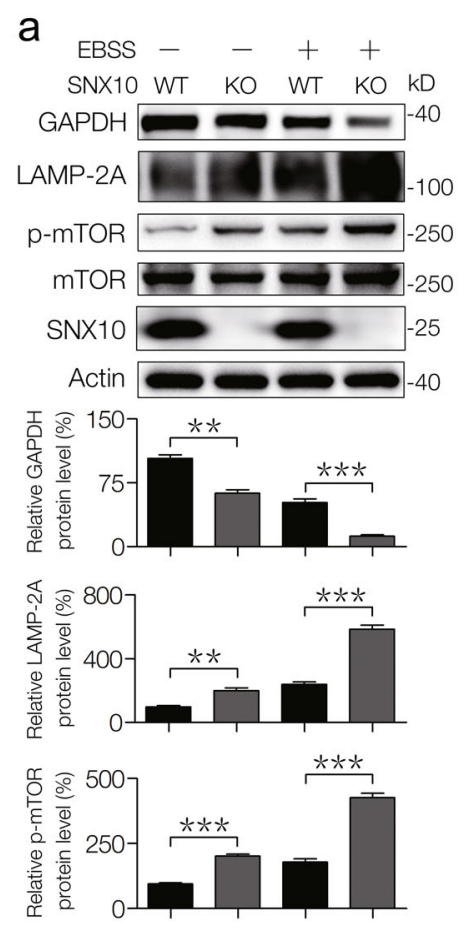

b

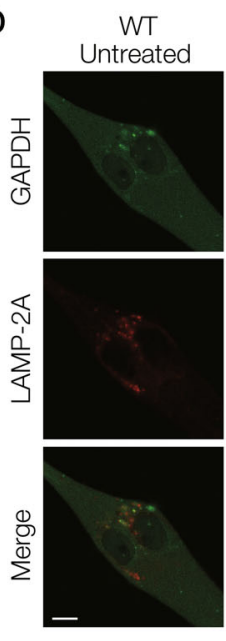

$\mathrm{KO}$

Untreated
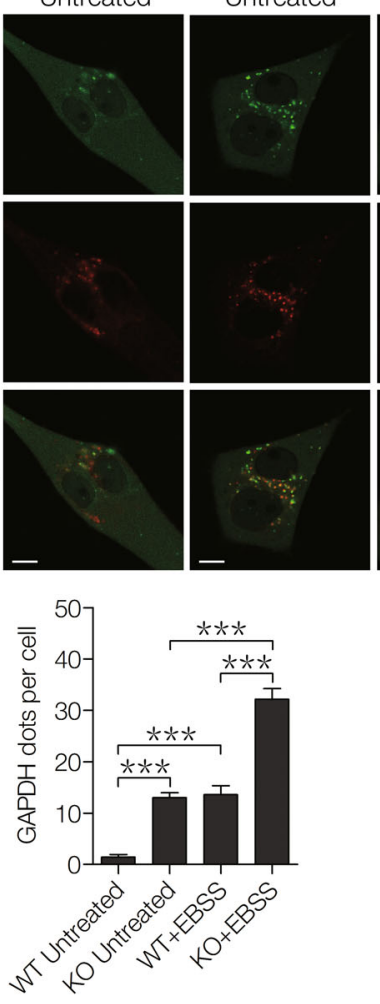

\section{C}

EBSS

SNX10 WT KO WT KO kD

$\mathrm{GAPDH}=-40$

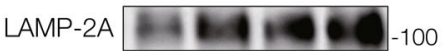

p-mTOR $\rightleftharpoons-250$

$\mathrm{mTOR}=-250$

SNX10

Actin

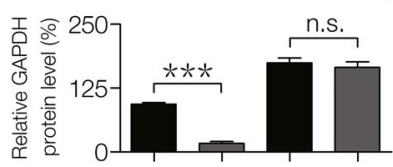

栾

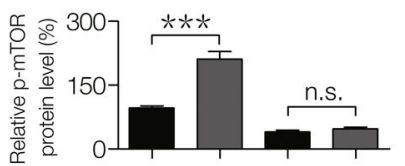

WT
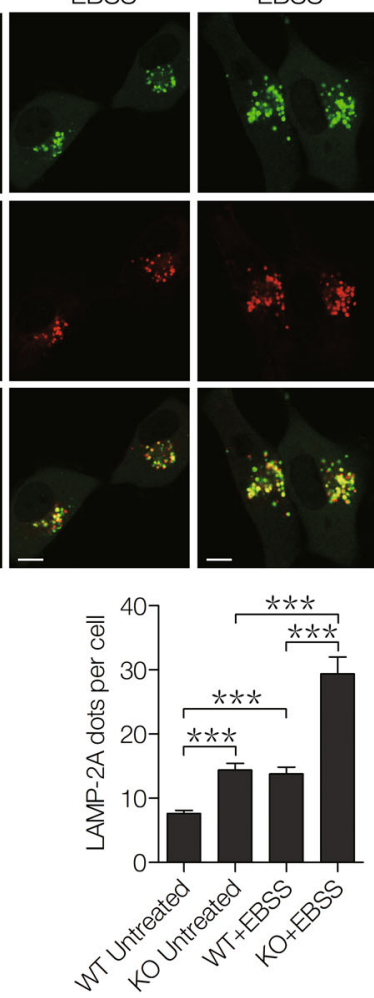

EBSS

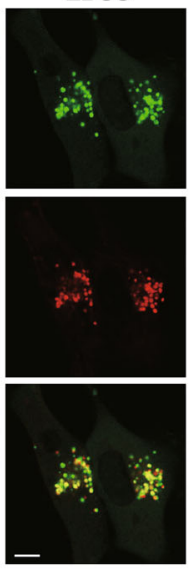

\section{d}
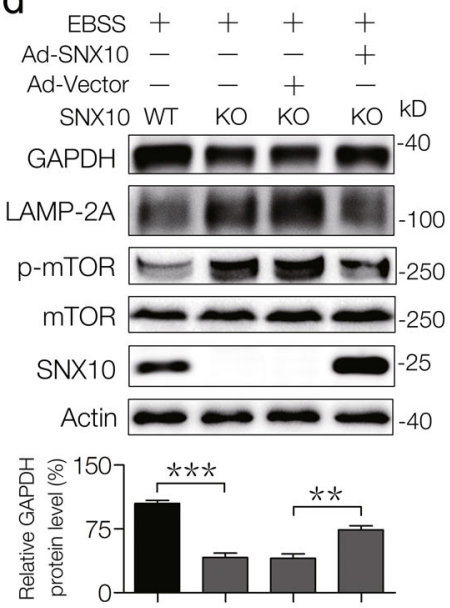

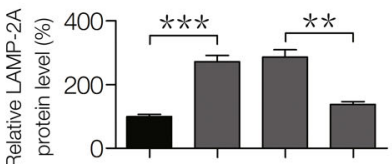

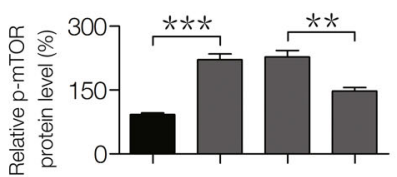

\section{e}

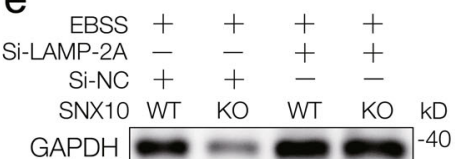

LAMP-2A $\square-100$

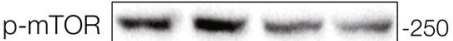

mTOR $=-250$

SNX10 $\longrightarrow-25$

Actin $\longrightarrow-40$
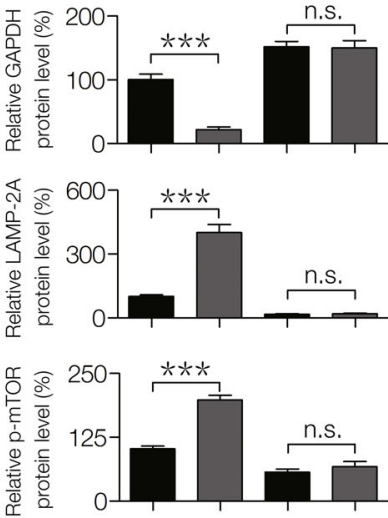

Fig. 5 (See legend on next page.) 
(see figure on previous page)

Fig. 5 Persistent activation of CMA degradation caused by SNX10 deficiency activates mTOR signaling. a Representative western blots showed the expression of indicated proteins in WT and SNX10 KO HCT116 cells with the treatment of EBSS for $24 \mathrm{~h}$. The bands of GAPDH, LAMP-2A, and p-mTOR were quantified using Quantity One software. b Co-staining of GAPDH and LAMP-2A in WT and SNX10 KO HCT116 cells treated with or without EBSS for $24 \mathrm{~h}$. Anti-GAPDH and anti-LAMP-2A antibodies were used to detect endogenous GAPDH and LAMP-2A, respectively. Scale bar (5 $\mu \mathrm{m})$. GAPDH- and LAMP-2A-positive puncta per cell from at least 200 cells in three independent experiments were quantified. c WT and SNX10 KO $\mathrm{HCT} 116$ cells were treated with EBSS and/or CQ for $24 \mathrm{~h}$, the total proteins were isolated and immunoblotted with indicated antibodies. The bands of GAPDH, LAMP-2A, and p-mTOR were quantified using Quantity One software. d WT, SNX10 KO HCT116 cells, and SXN10 KO HCT116 cells infected with Ad-Vector or Ad-SNX10 were treated with EBSS for $24 \mathrm{~h}$. LAMP-2A, GAPDH, and p-mTOR were detected by immunoblot, followed by densitometry quantification by Quantity One software. e WT and SNX10 KO HCT116 cells transfected with nontargeting siRNA (Si-NC) or LAMP-2A siRNA (Si-LAMP-2A) were treated with EBSS for $24 \mathrm{~h}$, the total proteins were isolated and immunoblotted with indicated antibodies. The bands of GAPDH, LAMP-2A, and p-mTOR were quantified using Quantity One software. Data are derived from at least three independent experiments and represented as means \pm SEM in the bar graphs. Not significant (n.s.), ${ }^{*} P<0.01$, and ${ }^{* *} P<0.001$

and differentiation. Our study also demonstrates that over-activated CMA by SNX10 deficiency was responsible for the upregulated amino-acid metabolism and mTOR signaling activation; eventually promoted the abnormal growth and proliferation of tumor cells.

mTOR signaling can integrate signals from growth factors, nutrients, mutagens, and hormones to induce cell proliferation, resistance to apoptosis, and autophagy ${ }^{21}$. It is frequently dysregulated in a variety of human cancers, including colorectal cancer ${ }^{22}$. K-RAS or PIK3CA mutation could activate mTOR signaling and promote CRC cell growth ${ }^{23}$. Clinically, mTOR manifests poor prognosis in stage II CRC patients ${ }^{24}$. Inhibition of mTOR expression results in decreased human CRC cell growth in vitro and in vivo ${ }^{25}$. Therefore, mTOR represent an attractive antitumor target in combination with strategies to target other pathways in $\mathrm{CRC}^{26}$.

Multiple upstream signaling inputs including amino acid participate in the regulation of mTOR activation ${ }^{27}$. mTOR comprises two complexes, termed mTORC1 and mTORC2. Only mTORC1 is controlled by metabolites, such as glucose and amino $\mathrm{acid}^{28}$. Lysosomes are key sites for mTORC1 activation that regulated by the small guanosine triphosphatases (GTPase) Rheb and Rag, which reside at the lysosomal surface where they function as regulator of the mTORC1 kinase activity ${ }^{29}$. A key event in amino-acid signaling is the Rag GTPase-mediated translocation of mTORC1 to the surface of lysosomes. Lysosomes sense amino-acid signaling via vacuolar $\mathrm{H}$ $(+)$-adenosine triphosphatase (V-ATPase) to activate mTORC1, which is known as the "inside-out" mechanism $^{30}$. Amino acids promote Rag to bind to Raptor (regulatory associated protein of mTOR) subunit of mTORC1 with consequent mTORC1 recruitment to lysosomes for its phosphorylation ${ }^{31}$. On the other hand, the dynamic of lysosomal amino acids is mTOR-dependent. Inhibition of mTOR strongly reduced the lysosomal efflux of most essential amino acids in nutrition starvation ${ }^{32}$. Due to its endosomal sorting and signaling functions, we consider
SNX10 could affect the activation process of amino-acid metabolites to mTOR signaling by regulating lysosomedependent processes, such as CMA.

Our recent study demonstrated SNX10 deficiency impaired the maturation of cathepsin A, which is a key enzyme involved in LAMP-2A degradation, thus increased CMA activity ${ }^{17}$. In the present study, we also confirmed that SNX10 KO upregulated LAMP-2A and CMA activity. CMA has high correlation with cancers. In contrast to the inhibition of macroautophagy in malignancy, upregulated CMA is required for cancer cell proliferation ${ }^{33}$. The interplay of CMA and macroautophagy has been reported in the literature. Induction of CMA compensates for the impairment of macroautophagy to promote hepatocellular carcinoma cell survival in the cirrhotic liver ${ }^{34}$. CMA-mediated anti-macroautophagic property is a key feature of tumorigenesis and metastasis in breast cancer ${ }^{35}$. Our recent study also showed that SNX10 KO inhibited macroautophagy, while increased CMA activity, promoting tumorigenesis and progression of colorectal cancer in male mice ${ }^{18}$. The connections between the metabolic changes and the development of cancer caused by CMA process is attracting more and more attention. CMA substrate proteins involved in the glycolysis, which contributes to the Warburg effect in cancer cells ${ }^{36}$. Metabolomics showed distinct effects of CMA and metabolic dysfunction, including increased branched-chain amino-acid levels, including Leu, Val, and Ile, in mice muscle and heart during aging ${ }^{37}$. CMA is also affected by ketone bodies, caloric restriction, and exercise in the context of neurodegenerative diseases ${ }^{38}$. Therefore, the influence of CMA on disease development is closely related to its role in cell catabolism and anabolism. However, to what extent CMA impact cell metabolism to promote $\mathrm{CRC}$ onset and progression remains largely unclear. CMA contributes to cellular QC through the removal of damaged or malfunctioning proteins ${ }^{39}$. Consequently, CMA has the potential to reshape the cell metabolism to promote cancer development. The elevated 


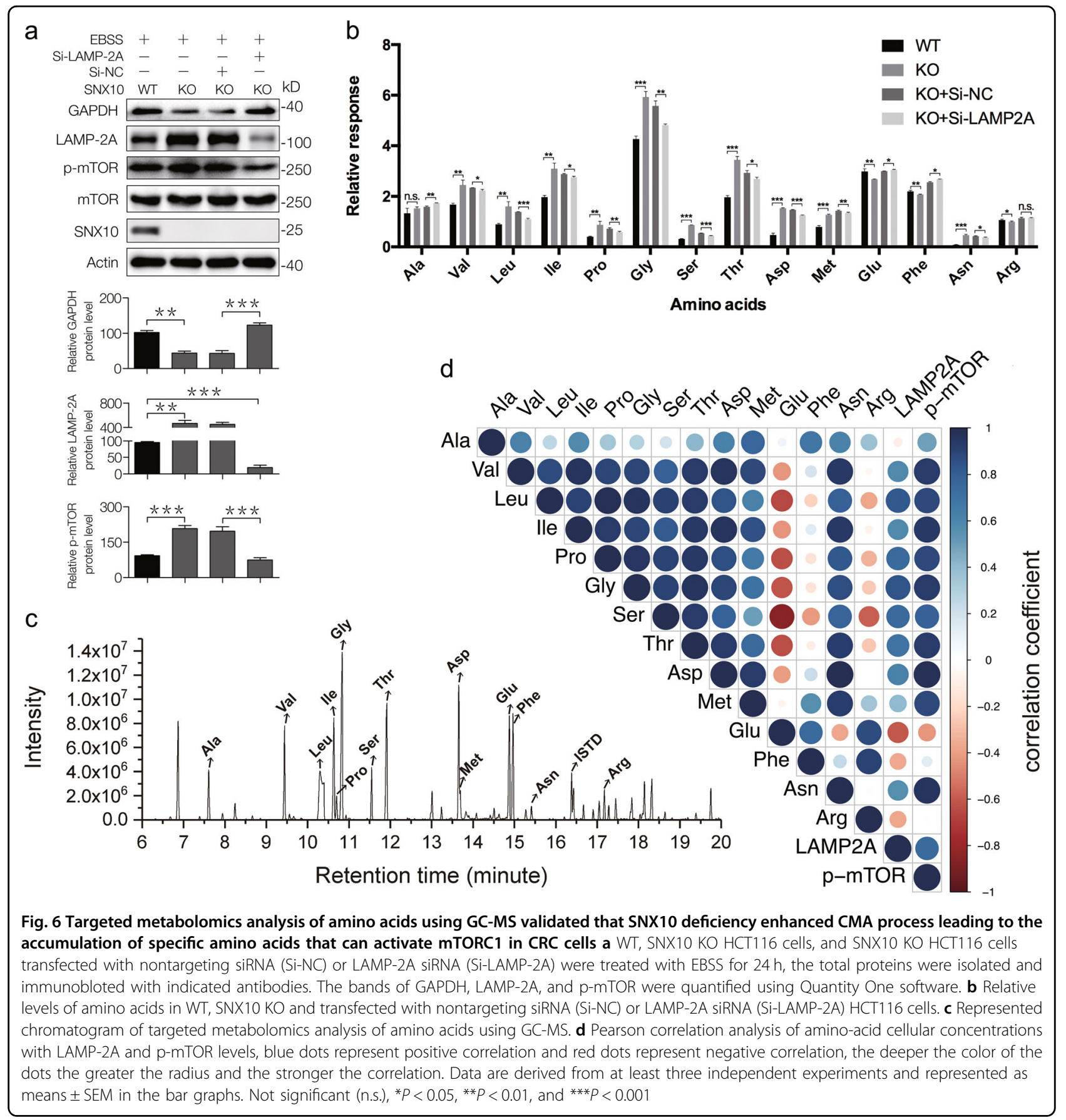

amino acids are not only the results of CMA substrate protein degradation but also act as cell signaling transductor to support tumorigenesis.

Given the close relationship between CMA and disease, its regulatory mechanism has aroused the interest of researchers. CMA process is mediated by binding to heat shock-cognate chaperone of $70 \mathrm{kDa}(\mathrm{HSC} 70)^{40}$. Then LAMP-2A is required to bind to the substrate proteins in lysosomes before the translocation can occur ${ }^{41}$. The substrate protein is ultimately degraded into amino acid by lysosome hydrolytic enzymes. Levels of LAMP-2A at the lysosomal membrane are limiting factor for $\mathrm{CMA}^{42}$ since it helps the lysosomal uptake of approximately $30 \%$ modified and oxidatively damaged soluble cytosolic proteins to transfer directly into the lumen of lysosomes for degradation ${ }^{43-45}$. Recently, LAMP-2A was found to be coupled with several diseases to CMA pathway, such as breast cancer ${ }^{46}$, Parkinson disease ${ }^{47}$, and hepatocellular carcinoma $^{48}$. Endosomes and lysosomes coordinate a balance of cellular materials for key cell processes. The 


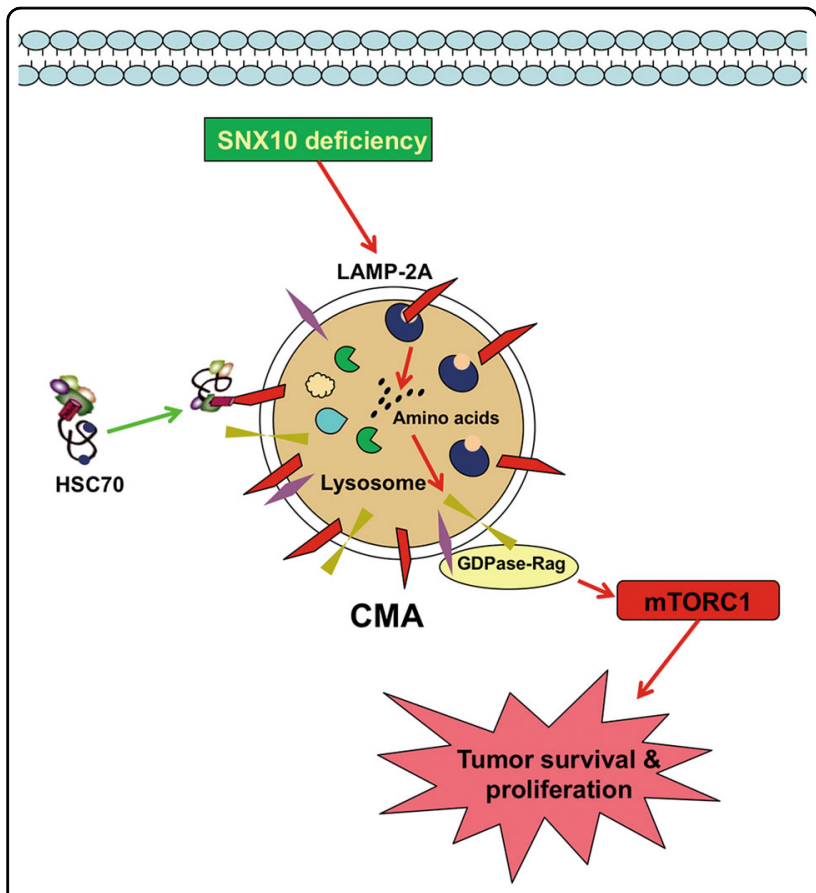

Fig. 7 Hypothetical regulatory mechanism of SNX10 on amino-acid metabolism through CMA pathway and its effect on tumor progression by $\mathrm{mTORC} 1$ activation

latest research showed that endoplasmic reticulum stressors lead to directly phosphorylation of LAMP-2A and CMA activation ${ }^{49}$. The present study confirmes that SNX10 deficiency enhanced CMA through the LAMP-2A mediated signaling in CRC tumor cells.

Present targeted metabolomics study on CRC cells revealed that CMA selectively upregulated the levels of amino acids that are indispensable for the activation of mTORC1. The levels of Val, Leu, Ile, Pro, Gly, Ser, Thr, Asp, Met, and Asn sharply increased in SNX10 KO cells compared with WT cells, thereafter decreased when CMA pathway was partially suppressed. This result suggests that CMA at least partially contributed to these specific amino-acid accumulation caused by SNX10 deficiency. Notably, all of these amino acids are essential for the activation of mTORC1 according to the literature ${ }^{19}$. The mechanisms of the amino-acid sensors and recognition in mTORC1 activation are still being identified. Several studies have explored the selectively regulating mechanism of mTOR activation by amino acids, especially Leu, Arg, and Gln, which are the most important amino acids in mTORC1 activation. Human member 9 of the solute carrier family 38 (SLC38A9) was proposed as a lysosomal membrane-resident protein competent of the amino-acid sensing machinery that controls the activation of mTORC1 ${ }^{50}$. SLC38A9 signals Arg sufficiency to mTORC1 activation $^{51}$. Gln flux regulates mTOR under a mechanism of bidirectional transporter SLC7A5/
SLC3A2 $2^{52}$. Study found that the Leu-binding capacity of Sestrin 2 is required for Leu to activate mTORC1 in cells $^{53}$. Therefore, the amino-acid regulatory mechanism of mTORC1 is different. In our study, instead of Arg and Gln, we found a group of amino acids that represented by Leu, Val, and Ile was closely correlated with SNX10/ $\mathrm{CMA} / \mathrm{mTOR}$ signaling. Whereas Arg showed no close correlation with mTOR phosphorylation, Glu and Phe even negatively connected with p-mTOR. Their concentrations were also decreased in SNX10 KO cells compared with WT cells. These results indicate that CMA process may be the unique mechanism on amino-acidmediated mTORC1 activation in SNX10-deficient cells. Our data suggest that SNX10 deficiency enhanced CMAmediated degradation of targeted proteins to increase specific amino acids, and subsequently activated mTORC1 to facilitate the tumor progression (Fig. 7).

In conclusion, our study showed that SNX10/CMA/ amino acid/mTOR axis promoted CRC progression in male FVB mice. SNX10 could regulate the amino-acid metabolism of CRC cells by controlling CMA pathway. The upregulated cellular specific amino acids activated mTORC1 to promote CRC tumorigenesis. Our study provides evidence for SNX10 as a potential target for CRC treatment.

\section{Materials and methods \\ Mice and CRC induction}

SNX10 KO homozygous and littermate mice background in FVB were transferred from the Institute of Development Biology and Molecular Medicine, Fudan University, Shanghai, China. Male SNX10 KO homozygous mice and their WT littermate mice aged 8-10 weeks were injected intraperitoneally with a single dose $(7.5 \mathrm{mg} / \mathrm{kg})$ of AOM followed by three cycles of $2.5 \%$ (weight/volume) DSS given in the drinking water for 5 days ${ }^{54}$.

\section{Chemicals, reagents, and antibodies}

Acetonitrile, methanol (HPLC grade), and dichloromethane were purchased from Sinopharm Chemical Reagent Company (Shanghai, China). Formic acid (HPLC grade) was purchased from Dima Technology (Lake Forest, CA, USA). And the standard chemicals for metabolite identification were obtained from Sigma-Aldrich (St. Louis, MO, USA) and Aladdin Industrial Corporation (Shanghai, China). Distilled water was filtered through a Milli-Q system (Millipore Corporation, Billerica, MA, USA). CQ (C6628), AOM (A5486), and EBSS (E2888) were purchased from Sigma-Aldrich. DSS $(36-50 \mathrm{kDa}$, 0216011080) was purchased from MP Biomedicals (Santa Ana, CA, USA). SNX10 (sc-104657) and GAPDH (sc47724) antibodies were the product of Santa Cruz Biotechnology (Dallas, TX, USA). Antibody against LAMP- 
2A (ab18528) was purchased from Abcam (Cambridge, UK). Antibody against $\beta$-actin (AA128) was from Beyotime Institute of Biotechnology (Haimeng, China). mTOR (2983) and p-mTOR (5536) antibodies were the product of Cell Signaling Technology (Boston, MA, USA).

\section{Tissue sample treatment and analysis by UHPLC-QTOF}

Tissue samples were treated using protein precipitation method. Agilent UHPLC system (Infinity 1260, Agilent Technologies, Santa Clara, CA, USA) equipped with a UPLC ACQUITY HSS T3 column (ACQUITY HSS T3, $2.1 \times 100 \mathrm{~mm}, 1.8 \mu \mathrm{m}$, Waters Corporation, Milford, MA, USA) were used for chromatographic separations. The UHPLC system was coupled to an Agilent 6520 electrospray ion source Accurate-Mass QTOF to obtain accurate mass time-of-flight data. For details, see Supplementary Experimental Procedures.

\section{Tissue sample treatment and analysis by GC-MS}

Tissue samples were treated using methyl silane derivatization. MS spectra of the sample extracts were acquired on the GC-MS analytical system with a 7890B GC and 5977A quadrupole mass analyzer in full scan mode. For details, see Supplementary Experimental Procedures.

\section{Untargeted metabolomics data processing and metabolic pathway analysis}

Raw mass spectra data were converted to mzDATA format files using Agilent MassHunter Qualitative Analysis (version B.06.00). Then the mzDATA files were uploaded to the XCMS Online (https://xcmsonline. scripps.edu) to preprocess to obtain the commaseparated value format peak intensity lists that contain $\mathrm{m} / \mathrm{z}$, retention time, and intensity of each peak. The further data statistic analysis was conducted by MetaboAnalyst 3.0 (http://www.metaboanalyst.ca) tool. The fully preprocessed data were analyzed with multivariate analysis methods of PCA and PLS-DA. Univariate analysis methods of FC analysis and $t$-test were also applied by the statistical analysis module of MetaboAnalyst.

Metabolic pathways and predicted metabolites in the pathways were analyzed utilizing mummichog 2.0 (http:// mummichog.org) at the command line level. Mummichog is a program written in python for analyzing data from high-throughput, untargeted high-resolution LC-MS metabolomics, bypassing the tedious and challenging metabolite identification ${ }^{55}$. For GC-MS data, the feature ions with average variable importance in the projection (VIP) values of PLS-DA components higher than 1 (VIP > 1) were selected as discriminating features of further identification. The raw mass spectra data were imported to MassHunter for mass spectra deconvolution and metabolites identification by comparation of the $\mathrm{m} / \mathrm{z}$ values with NIST databases. The compound with highest matching score was identified to the $\mathrm{m} / \mathrm{z}$ value. For details, see Supplementary Experimental Procedures.

\section{Cell culture and transfection}

NCM460, HCT116, Caco-2, and SW480 cells were purchased from the American Type Culture Collection (Manassas, VA, USA). Cells were cultured in Dulbecco's modified Eagle's (Gibco, Thermo Fisher Scientific, Waltham, MA, USA) supplemented with $10 \%$ fetal bovine serum (Biological Industries, Beit Haemek, Israel) at $37^{\circ} \mathrm{C}$ under $5 \%$ (volume/volume) $\mathrm{CO}_{2}$ atmosphere. LAMP-2A siRNA was transfected into cells by Lipofectamine RNAi max (Invitrogen, Thermo Fisher Scientific, Waltham, MA, USA) for $72 \mathrm{~h}$. The siRNA sequences are as follows: LAMP-2A siRNA: GGCAGGAGUACUUAUUCUAGU; nontargeting siRNA: AATTCTCCGAACGTGTCACGT.

\section{Immunofluorescence staining}

After treatment, cells were fixed in $4 \%$ formaldehyde for $10 \mathrm{~min}$ at ambient temperature, followed by permeabilization and blocking with phosphate buffer solution (PBS) containing $10 \%$ normal goat serum and $0.1 \%$ saponin (Sigma-Aldrich). Cells were then incubated with primary antibodies for $2 \mathrm{~h}$ at ambient temperature. After washing three times with PBS, cells were incubated with appropriate Alexa Fluor-labeled secondary antibodies (1:500, Invitrogen, in blocking buffer) for $1 \mathrm{~h}$ at ambient temperature. The coverslips were mounted in Prolong Gold anti-fade reagent with 4',6-diamidino-2-phenylindole (Invitrogen) and inspected with a confocal microscope (Zeiss 710, Zeiss Group, Oberkochen, Germany).

\section{Histological analysis}

Mice colorectum were removed, fixed with formaldehyde solution, and sectioned routinely into $4-5 \mu \mathrm{m}$ slabs, which were stained with hematoxylin and eosin, followed by analysis of tumor size using a light microscope.

\section{Cell proliferation assay}

Cell proliferation was tested using BrdU ELISA kit according to the manufacturer's instructions (Exalpha Biologicals, Shirley, MA, USA).

\section{Colony formation assay}

WT and SXN10 KO cells were plated in six-well plates at approximately 200 cells/well in $2 \mathrm{ml}$ of complete media. For SNX10 reintroduction group, SXN10 KO cells were infected with Adenovirus-vector or Adenovirus-SNX10 once a week. The assay should be stopped when the colonies are clearly visible even without looking under microscope. Cells then were stained with $0.1 \%$ crystal violet and number of colonies was counted. 


\section{Western blot}

Western blot analysis was performed as we described previously $^{56}$.

\section{Cell targeted metabolomics by GC-MS}

The medium was discarded and cells were washed with $10 \mathrm{ml}$ ambient-temperature PBS (BasalMedia, China). Then, the plates were quenched by directly adding $15 \mathrm{ml}$ of liquid nitrogen. The plates were transferred to a $-80{ }^{\circ} \mathrm{C}$ freezer until extracted and assayed. For metabolites extraction, $1.2 \mathrm{ml}$ of ice-cold methanol containing $20 \mu \mathrm{g} / \mathrm{ml}$ tridecanoic acid (Sigma-Aldrich) as ISTD was added to each plate and cells were detached from the surface with a disposable cell scraper (Crystalgen, China) and collected into a centrifuge tube for extraction. Cell lysates were then prepared with $30 \mathrm{~min}$ ultra-sonication. After centrifugation at 12000 rounds/ min for $10 \mathrm{~min}$, supernatant was transferred and dried under the nitrogen stream at $40{ }^{\circ} \mathrm{C}$. The subsequent derivatization procedures were as same as the untargeted metabolomics experiment. After derivatization, the supernatants were analyzed on the GC-MS analytical system with a 7890B GC and 5977A quadrupole mass analyzer (Agilent) in selected ion monitor mode. For details, see Supplementary Experimental Procedures.

\section{Pearson correlation coefficient computation}

The Pearson correlation coefficient is calculated by the $\mathrm{R}$ language program. For details, see Supplementary Experimental Procedures.

\section{Statistics}

Data are presented as mean \pm SEM, Statistical analysis were performed by GraphPad Prism version 6.0. Unpaired two-tailed Student's $t$-test or one-way analysis of variance was used to compare the mean values of the groups. Statistical significance was accepted at $P<0.05$.

\section{Acknowledgements}

This study was funded by National Natural Science Foundation of China (No. 81773744 and 81573441).

\section{Conflict of interest}

The authors declare that they have no conflict of interest.

\section{Publisher's note}

Springer Nature remains neutral with regard to jurisdictional claims in published maps and institutional affiliations.

Supplementary Information accompanies this paper at (https://doi.org/ 10.1038/s41419-018-0719-2).

Received: 26 January 2018 Revised: 16 May 2018 Accepted: 17 May 2018 Published online: 04 June 2018

\section{References}

1. Torre, L. A. et al. Global cancer statistics, 2012. CA Cancer J. Clin. 65, 87-108 (2015).

2. Brenner, H., Kloor, M. \& Pox, C. P. Colorectal cancer. Lancet 383, 1490-1502 (2014).

3. American Cancer Society. Cancer facts and figures 2015. https://www.cancer. org/acs/groups/content/@editorial/documents/document/acspc-044552.pdf (2015).

4. Nappi, A. et al. Metastatic colorectal cancer: role of target therapies and future perspectives. Preprint at https://doi.org/10.2174/ 1568009617666170209095143 (2017).

5. Williams, C., DiLeo, A., Niv, Y. \& Gustafsson, J. A. Estrogen receptor beta as target for colorectal cancer prevention. Cancer Lett. 372, 48-56 (2016).

6. Gil, J., Pesz, K. A. \& Sasiadek, M. M. May autophagy be a novel biomarker and antitumor target in colorectal cancer? Biomark. Med. 10, 1081-1094 (2016).

7. Seyfried, T. N., Flores, R. E., Poff, A. M. \& D'Agostino, D. P. Cancer as a metabolic disease: implications for novel therapeutics. Carcinogenesis 35, 515-527 (2014).

8. Wishart, D. S. Emerging applications of metabolomics in drug discovery and precision medicine. Nat. Rev. Drug Discov. 15, 473-484 (2016).

9. Uchiyama, K. et al. Serum metabolomics analysis for early detection of colorectal cancer. J. Gastroenterol. 52, 677-694 (2017).

10. Farshidfar, F. et al. A validated metabolomic signature for colorectal cancer exploration of the clinical value of metabolomics. Br. J. Cancer 115, 848-857 (2016).

11. Goedert, J. J. et al. Fecal metabolomics: assay performance and association with colorectal cancer. Carcinogenesis 35, 2089-2096 (2014).

12. Cullen, P. J. \& Korswagen, H. C. Sorting nexins provide diversity for retromerdependent trafficking events. Nat. Cell Biol. 14, 29-37 (2011).

13. Nagelkerke, A., Sweep, F. C., Geurts-Moespot, A., Bussink, J. \& Span, P. N. Therapeutic targeting of autophagy in cancer. Part I: molecular pathways controlling autophagy. Semin. Cancer Biol. 31, 89-98 (2015).

14. Mizushima, N., Levine, B., Cuervo, A. M. \& Klionsky, D. J. Autophagy fights disease through cellular self-digestion. Nature 451, 1069-1075 (2008).

15. Suzuki, J., Nakajima, W., Suzuki, H., Asano, Y. \& Tanaka, N. Chaperone-mediated autophagy promotes lung cancer cell survival through selective stabilization of the pro-survival protein, MCL1. Biochem. Biophys. Res. Commun. 482, 1334-1340 (2017).

16. Tasset, I. \& Cuervo, A. M. Role of chaperone-mediated autophagy in metabolism. FEBS J. 283, 2403-2413 (2016).

17. You, Y. et al. SNX10 mediates alcohol-induced liver injury and steatosis by regulating the activation of chaperone-mediated autophagy. Preprint at https://doi.org/10.1016/j.jhep.2018.01.038 (2018).

18. Zhang, S. et al. Sorting nexin 10 acts as a tumor suppressor in tumorigenesis and progression of colorectal cancer through regulating chaperone mediated autophagy degradation of p21(Cip1/WAF1). Cancer Lett. 419, 116-127 (2018).

19. Dyachok, J., Earnest, S., Iturraran, E. N., Cobb, M. H. \& Ross, E. M. Amino Acids Regulate mTORC1 by an Obligate Two-step Mechanism. J. Biol. Chem. 291, 22414-22426 (2016).

20. Pavlova, N. N. \& Thompson, C. B. The emerging hallmarks of cancer metabolism. Cell. Metab. 23, 27-47 (2016).

21. Weijenberg, M. P. et al. The mTOR pathway and the role of energy balance throughout life in colorectal cancer etiology and prognosis: unravelling mechanisms through a multidimensional molecular epidemiologic approach. Curr. Nutr. Rep. 2, 19-26 (2013)

22. Ekstrand, A. I., Jonsson, M., Lindblom, A., Borg, A. \& Nilbert, M. Frequent alterations of the PI3K/AKT/mTOR pathways in hereditary nonpolyposis colorectal cancer. Fam. Cancer 9, 125-129 (2010).

23. Kim, A. et al. Coexistent mutations of KRAS and PIK3CA affect the efficacy of NVP-BEZ235, a dual PI3K/MTOR inhibitor, in regulating the PI3K/MTOR pathway in colorectal cancer. Int. J. Cancer 133, 984-996 (2013).

24. Cai, Z. et al. Significance of mTOR signaling and its inhibitor against cancer stem-like cells in colorectal cancer. Ann. Surg. Oncol. 21, 179-188 (2014).

25. Zhang, Y. J. et al. mTOR signaling pathway is a target for the treatment of colorectal cancer. Ann. Surg. Oncol. 16, 2617-2628 (2009).

26. Kim, D. D. \& Eng, C. The promise of mTOR inhibitors in the treatment of colorectal cancer. Expert Opin. Investig. Drugs 21, 1775-1788 (2012).

27. Ma, X. J. M. \& Blenis, J. Molecular mechanisms of mTOR-mediated translational control. Nat. Rev. Mol. Cell Biol. 10, 307-318 (2009).

28. Jewell, J. L. \& Guan, K. L. Nutrient signaling to mTOR and cell growth. Trends Biochem. Sci. 38, 233-242 (2013). 
29. Bar-Peled, L. \& Sabatini, D. M. Regulation of mTORC1 by amino acids. Trends Cell Biol. 24, 400-406 (2014).

30. Zoncu, R. et al. mTORC1 senses lysosomal amino acids through an inside-out mechanism that requires the vacuolar H+-ATPase. Science 334, 678-683 (2011).

31. Bar-Peled, L. et al. A tumor suppressor complex with GAP activity for the Rag GTPases that signal amino acid sufficiency to mTORC1. Science $\mathbf{3 4 0}$, 1100-1106 (2013).

32. Abu-Remaileh, M. et al. Lysosomal metabolomics reveals V-ATPase- and mTOR-dependent regulation of amino acid efflux from lysosomes. Science 358, 807-813 (2017)

33. Kon, M. et al. Chaperone-mediated autophagy is required for tumor growth. Sci. Transl. Med. 3, 109ra117 (2011).

34. Chava, S. et al. Chaperone-mediated autophagy compensates for impaired macroautophagy in the cirrhotic liver to promote hepatocellular carcinoma. Oncotarget 8, 40019-40036 (2017).

35. Han, Q. et al. Downregulation of ATG5-dependent macroautophagy by chaperone-mediated autophagy promotes breast cancer cell metastasis. Sci. Rep. 7, 4759 (2017).

36. Tang, Y. et al. Chaperone-mediated autophagy substrate proteins in cancer. Oncotarget 8, 51970-51985 (2017).

37. Zhou, J. et al. Changes in macroautophagy, chaperone-mediated autophagy, and mitochondrial metabolism in murine skeletal and cardiac muscle during aging. Aging (Albany NY) 9, 583-599 (2017).

38. Loos, B., Klionsky, D. J. \& Wong, E. Augmenting brain metabolism to increase macro- and chaperone-mediated autophagy for decreasing neuronal proteotoxicity and aging. Prog. Neurobiol. 156, 90-106 (2017).

39. Cuervo, A. M. \& Wong, E. Chaperone-mediated autophagy: roles in disease and aging. Cell Res. 24, 92-104 (2014).

40. Cuervo, A. M. Chaperone-mediated autophagy: selectivity pays off. Trends Endocrinol. Metab. 21, 142-150 (2010).

41. Cuervo, A. M. \& Dice, J. F. A receptor for the selective uptake and degradation of proteins by lysosomes. Science 273, 501-503 (1996).
42. Cuervo, A. M. \& Dice, J. F. Regulation of lamp2a levels in the lysosomal membrane. Traffic 1, 570-583 (2000).

43. Zhou, D. L. et al. Lamp-2a facilitates MHC class II presentation of cytoplasmic antigens. Immunity 22, 571-581 (2005).

44. Massey, A., Kiffin, R. \& Cuervo, A. M. Pathophysiology of chaperone-mediated autophagy. Int. J. Biochem. Cell Biol. 36, 2420-2434 (2004).

45. Majeski, A. E. \& Dice, J. F. Mechanisms of chaperone-mediated autophagy. Int. J. Biochem. Cell Biol. 36, 2435-2444 (2004).

46. Saha, T. LAMP2A overexpression in breast tumors promotes cancer cell survival via chaperone-mediated autophagy. Autophagy 8, 1643-1656 (2012).

47. Xilouri, M., Brekk, O. R., Kirik, D. \& Stefanis, L. LAMP2A as a therapeutic target in Parkinson disease. Autophagy 9, 2166-2168 (2013).

48. Ding, Z. B. et al. Lamp2a is required for tumor growth and promotes tumor recurrence of hepatocellular carcinoma. Int. J. Oncol. 49 2367-2376 (2016).

49. Li, W. et al. Phosphorylation of LAMP2A by p38 MAPK couples ER stress to chaperone-mediated autophagy. Nat. Commun. 8, 1763 (2017).

50. Rebsamen, M. et al. SLC38A9 is a component of the lysosomal amino acid sensing machinery that controls mTORC1. Nature 519, 477-481 (2015).

51. Wang, S. Y. et al. Lysosomal amino acid transporter SLC38A9 signals arginine sufficiency to mTORC1. Science 347, 188-194 (2015).

52. Nicklin, P. et al. Bidirectional transport of amino acids regulates mTOR and autophagy. Cell 136, 521-534 (2009).

53. Wolfson, R. L. et al. METABOLISM Sestrin2 is a leucine sensor for the mTORC1 pathway. Science 351, 43-48 (2016).

54. Neufert, C., Becker, C. \& Neurath, M. F. An inducible mouse model of colon carcinogenesis for the analysis of sporadic and inflammation-driven tumor progression. Nat. Protoc. 2, 1998-2004 (2007).

55. Li, S. et al. Predicting network activity from high throughput metabolomics. PLoS Comput. Biol. 9, e1003123 (2013).

56. Zhou, C. et al. BIG1, a brefeldin A-inhibited guanine nucleotide-exchange protein regulates neurite development via PI3K-AKT and ERK signaling pathways. Neuroscience 254, 361-368 (2013). 